\title{
Two “Unusual” Cerebrovascular Risk Factors: A Not- Easy Assessment. The Opinion of The Cardiogeriatric Physician
}

\section{Valerio Massimo Magro ${ }^{1 *}$ and Walter Verrusio ${ }^{2}$}

${ }^{1}$ Department of Internal Medicine and Geriatry, Second University of Naples, Italy

${ }^{2}$ Department of Cardiovascular, Respiratory, Nephrological, Anesthesiological and Geriatric Sciences, Sapienza University of Rome, Italy

*Corresponding author: Valerio Massimo Magro, Department of Internal Medicine and

Geriatry, Second University of Naples, Italy.

Received Date: October 16, 2018

Published Date: October 30, 2018

\section{Background}

There are still controversies regarding the embolic potential of certain conditions in which the clinician and/or the geriatrician may encounter, even randomly, during the assessment of the requirements, examined effort for the prevention of recurrent stroke/TIA/cryptogenic stroke. We take a cue from two cases received for our observation for the self-audit tariff and launch, if possible, some food for thought.

\section{Case Report 1}
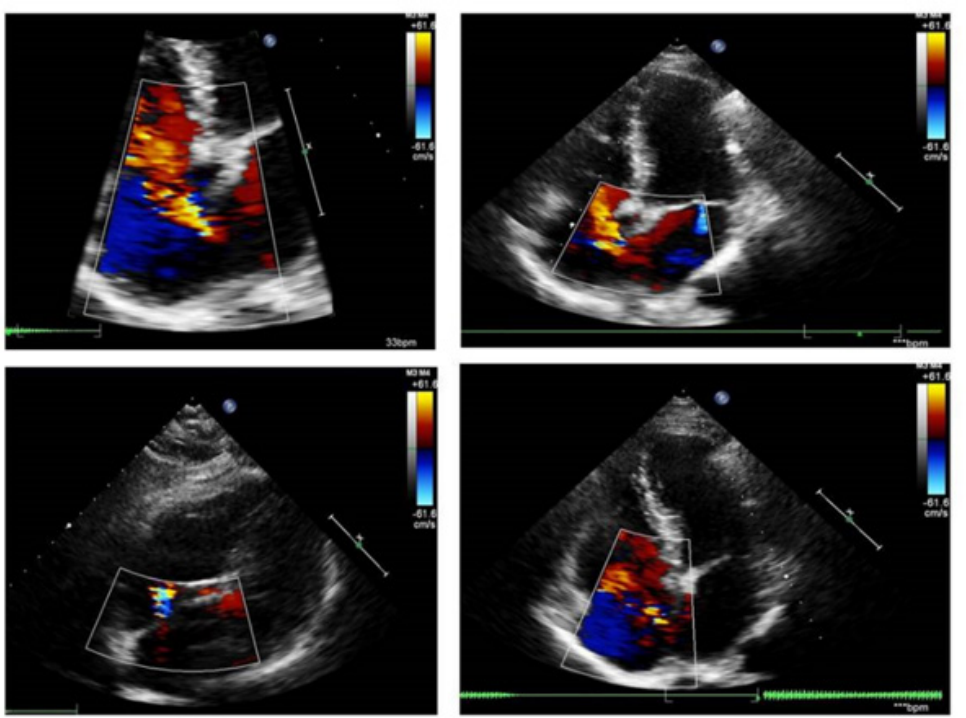

Figure 1: Transthoracic echocardiogram. Four-chamber apical window showing the presence of aneurysm of the interatrial septum. The colordoppler study reveals the presence of shunts directed to the right heart.

A 48-year-old female patient, former smoker. We found a hypoechoic nodular formation specimen with speculated margins, with a $12 \mathrm{~mm}$ major axis, identified on the screening radiograph. Next FNAB (BIRADS 5) and diagnosis by the echo-assisted core biopsy of right "no special type" infiltrating breast cancer undergoing to a superior right quadrantectomy and ipsilateral lymphadenectomy. At histological examination, presence of moderate differentiation, vascular emboli and perineural infiltration; staging, sec. Elton- 
Ellis pT1-G2; N0; Mx. Immunohistochemical profile: ER 90\%, PGR 80\%, Ki67 5\%; HER2 score 3+. The patient started on therapy with cyclophosphamide $960 \mathrm{mg}$, eirenicon $144 \mathrm{mg}$, dexamethasone $12 \mathrm{mg}$, pantoprazole $20 \mathrm{mg}$, ondansetron $8 \mathrm{mg}$, folia $5 \mathrm{mg}$, paracetamol $1 \mathrm{~g}$, fosaprepitant meglumine $150 \mathrm{mg}$, peg filgrastim $6 \mathrm{mg}$. At the control, discrete general conditions (with a slight anamnestic dyspnea), good vital signs and blood tests, EKG trace in the limits. At the transthoracic echocardiogram, "normal atrial (atrial diameter $3.2 \mathrm{~cm}$-right atrial area: $16.2 \mathrm{~cm} 2$ ) and left and right ventricular dimensions, norm kinesis and good ejection fraction (EF > 50\%); mitral-tricuspid and pulmonary insufficiency were shown. PAPs approx. 50mm Hg. Normal caliber of lower cava vein $(1.7 \mathrm{~cm})$, collapsed with breath acts. An aneurysm of the interatrial septum (IAS) was also found with significant patency of the IAS and a left-right shunt (Figure 1). After collegial review, we decided to send the patient to perform the test with the microbubbles for a better definition of the case and the decision of a possible interventional or pharmacological therapy (anticoagulant), the latter being preferred.

\section{Case Report 2}

A 66-year-old male patient, normal weight, reached for a presence of a severe degree of gait disorder, for whom the patient had previously taken leva-dopa/benderizine, therapy suspended for absent improvements and incipient appearance of drowsiness, and in current therapy with selegiline $5 \mathrm{mg}$ and pramipexole 0.9mg. Vital signs were within limits except PA 160/90mmHg. The Multidimensional Assessment (MDA) also highlighted an important cognitive disturbance with the Mental Mental State Examination (MMSE) score of 24/30, Activity Daily Living (ADL) 6/6 and Instrumental Activity Daily Living (IADL) $7 / 8$, with a presence of a slight depressive component highlighted at the Geriatric Depression Scale (GDS) with 15 items. At the examination of the mowing presence of a mowing gait on the left with the risk of falling into retropulsion (positiveness of the Tinetti scale due to high falls risk) and positiveness of the Magazine's sign on the left also for the ipsilateral upper limb. Within the limits the blood chemistry tests. The carotid ecocolordoppler exam revealed an increased and widespread my intimal thickness at the level of all arterial axes bilaterally explored with the presence of bulbar fibrocalcific plaque on the right and the presence of a stenosis about $25 \%$ and likewise on the left with stenosis about $30 \%$, without alterations of the velocimetry. Artery patency of the subclavian and vertebral arteries. Brain magnetic resonance imaging (MRI) showed "at the level of adjacent white matter of the left frontal horn (at the rostrum of the corpus callosum) presence of an hyperintense image in T2 and FLAIR images, with diffusion shrinkage (evolving ischemic vascular lesion), presence of multiple gliotic foci on a chronic hypoperfusion basis at the periventricular white matter, semi vial centers and external capsules. Ischemic lacunar outcomes at the lower portion of the right cerebellar hemisphere, at the bridge and in bilateral lentil-capsular sites. Peri cerebral and peri cerebellar subarachnoid spaces slightly larger for cortical atrophy". An echocardiogram with associated study of the aorta was performed, looking for emboli gene sources: "left ventricle of normal size with conserved global contractility $(\mathrm{EF}>50 \%)$, mild hypertrophy of the interventricular septum. Left atrium of normal size for body surface area (Vol LA/ BSA $22.5 \mathrm{ml} / \mathrm{sqm}$ ). Ectasia aortic root with sclerocalcifications of the cuspids, normal caliber of the aortic arch, with a lowered fibrocalcific formation in the aortic lumen on the inferior wall (Figure 2). Normal abdominal aorta in the explored features". This finding was confirmed in the Angio-CT with contrast. Then, therapy was modified: omeprazole $20 \mathrm{mg}$, perindopril/amlodipine $10 / 5 \mathrm{mg}$ (based on the PROGRESS trial), Neuropore® (choline, alpha-lipoic acid, vitamin $\mathrm{C}$ and $\mathrm{E}$ supplement), warfarin according to INR, selegiline $10 \mathrm{mg}$, simvastatin $10 \mathrm{mg}$.

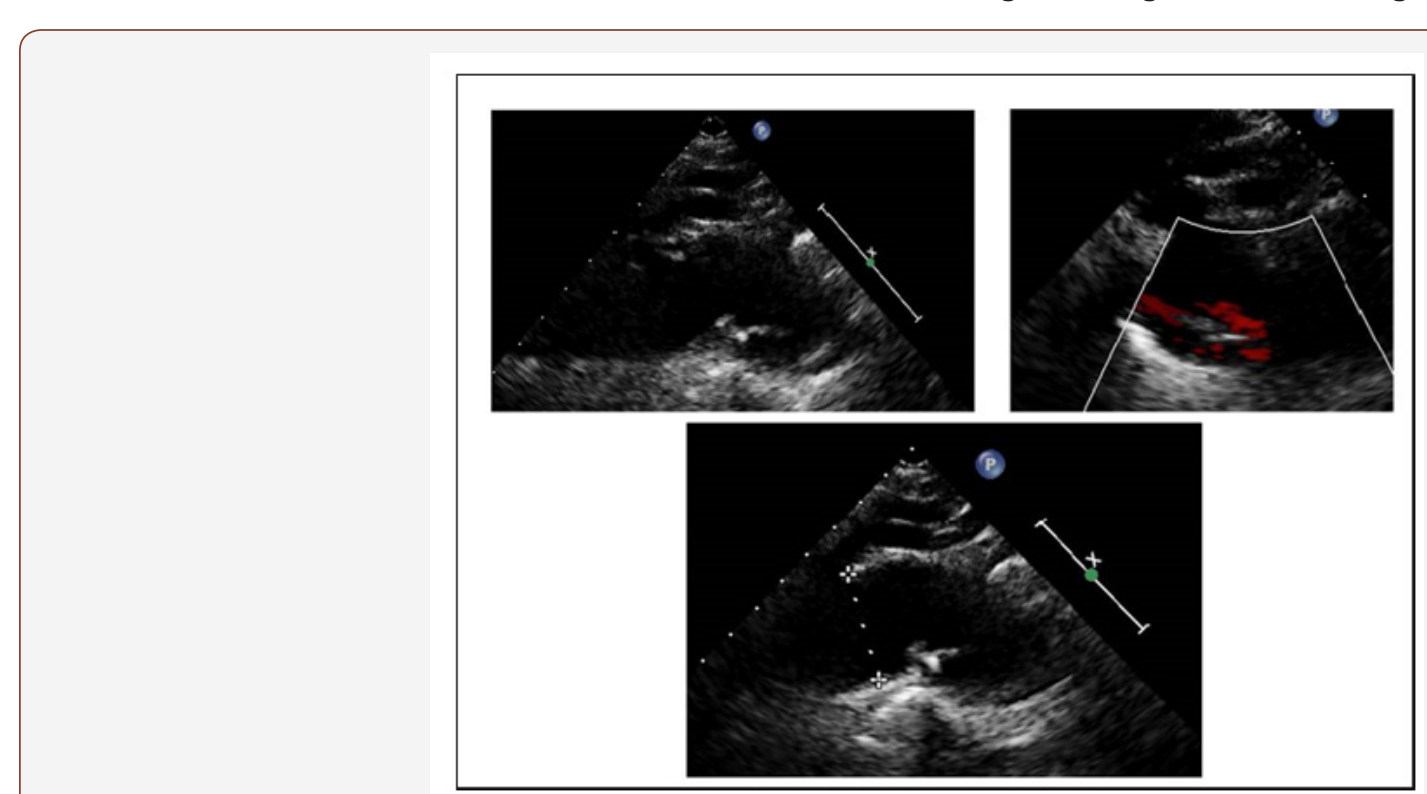

Figure 2: Transthoracic echocardiograms of the aortic arch showing a protruding atherosclerotic plaque with a mobile projection.

\section{Discussion}

In several stroke cases it is not possible to establish the etiology of the event. However, in the last decade, with the implementation of ultrasound imaging methods, in some patients with classified stroke of uncertain origin, it has been possible to reveal the existence of different conditions that, following in prospective and 
retrospective studies, have been shown to have a cerebrovascular risk.

The aneurysm of the IAS is a thinning of the wall of the IAS, sometimes associated with the persistence of the foramen oval (PFO), which tends to move between the atria in systole. It is a cardiac anomaly diagnosed with echocardiography and whose presence is often described in literature as associated with stroke (SPARC trial) [1]. Although literature has investigated the topic, there are doubts on the pathogenesis of stroke from potentially source of emboli linked to this condition [2]. The patient in question, in addition, was a cancer patient. The interrelation between stroke and cancer is complex. In fact, both can occur independently in each patient or the cancer can lead directly or indirectly to a stroke through various and heterogeneous mechanisms, including hypercoagulability, non-bacterial endocarditis and adverse treatment events. In this already complex mosaic the presence of a congenital heart disease can be inserted, which, also through the mentioned mechanisms, can generate repercussions on the intracardiac hemodynamics potentially source of cerebral emboli $[3,4]$. A large-scale metaanalysis of Overall JR et al has confirmed, however, that both aneurysm of the IAS and PFO are significantly associated with stroke in young adult patients (aged <55) [5]. Although there are official and national [6]. and international [7]. consensus documents, there is no such evidence in the literature to recommend one course of action rather than another, although the increasing use of devices pushes the therapeutic choices towards this direction $[8,10]$. However, we believe that a decision of this kind on the one hand presupposes an assessment of the thrombotic and embolic risk that takes into account, in addition to the anomaly itself and the extent of the shunt, the presence of valvopathies and atrial anatomy and the presence of any systolic and diastolic dysfunction of them (in this evaluation the speckle tracing appears to be a very interesting and precise technique compared to the existing ultrasonographic ones), on the other hand a more extensive evaluation of the patient, in a geriatric or multidimensional one, with a MDA that also includes other items (such as the Karnowski score). In the decision-making of this case we tried to stick to the conclusions of the study by Cerrato P et al. [11]. despite the low case studies studied (86 patients) and without any of these patients suffering from oncological pathology. Therefore, a number of further trials on more extensive case studies are needed to determine the optimal treatment of patients, with particular reference to the selection of patients sub-patients also affected by neoplasms in order not only to confirm whether the aneurysm of IAS (isolated or not) is a direct cause of cerebral or concurrent ischemia or an additional risk factor of cryptogenic stroke in these patients, but also which is the optimal therapy in cancer patients.

The same doubts concern atheroma's located at the level of the aortic arch [12-14]. Although aortic arch atheromasia is a common finding in the elderly population, there are not only case reports [1517]. and case series [18]. that have documented their emollitions capacity, but also several trials (for example, the French Study of Aortic Plaques in Stroke study) and reviews in Literature shown the strong association between ischemic stroke and this finding, especially if plaque occurs with mobile and floating components $[19,20]$. Despite the improvement of technologies, the extensive use of imaging techniques and the advent of new parameters to assess the dangers of these plaques [21,23]. the overall picture of risk definition, optimal therapy and follow up of these patients remain subject of discussion [24-26]. Moreover, compared to a low number of studies, even under-sampled, there is no unanimous opinion even on the best prophylactic strategy (even the recent trial Aortic Arch Related Cerebral Hazard ARCH does not provide an exhaustive answer) [27]. In these cases, the acetylsalicylic acid alone may not be of adequate protection (hence the use of anticoagulant therapy) towards new ischemic cerebrovascular events. However, in front of trials that have directed attention to a more potent associated anti-aggregation (CHARISMA trial, MATCH trial), the use of the anticoagulation drugs remains a very controversial option, as a potential source of clinical worsening, cerebral hemorrhage/ hemorrhagic stroke or the same plaque (with thrombotic overlap) or even origin to systemic embolism.

\section{Conclusion}

The aneurysm of the IAS and the atheroma of the aortic arch undoubtedly represent factors that increase the risk of stroke. However, these factors can be modified with appropriate nonpharmacological or pharmacological strategies. Currently, with the easy diagnosis they are facing, even for the increasingly widespread use of bloodless technologies (like the ultrasonography) there is a growing interest in the best strategies to be adopted. In fact, the role of these conditions appears to be currently not fully documented either as an independent risk factor but also about the best therapeutic and follow-up strategy. Pending further trials, increasingly targeted and with the significant patient cohorts, it seems reasonable not to exempt themselves from a careful evaluation of the individual patient using a clinical and multidimensional method and with a definitive team judgment among the different branches of specialists involved (geriatrician, cardiologist, neurologist, radiologist, oncologist, general practitioner).

\section{Acknowledgement}

None.

\section{Conflict of Interest}

No Conflict of Interest.

\section{References}

1. Mas JL, Arquizan C, Lamy C, Zuber M, Cabanes L, et al. (2001) Recurrent cerebrovascular events associated with patent foramen ovale, atrial septal aneurysm, or both. N Engl J Med 345(24): 1740-1746.

2. Doufekias E, Segal AZ, Kizer JR (2008) Cardiogenic and aortogenic brain embolism. J Am Coll Cardiol 51(11): 1049-1059.

3. Carerj S, Zito C, Oliva S, Tassone G, Luzza F, et al. (2005) Aneurisma del setto interatriale: reale sorgente cardioembolica? Ital Heart J Suppl 6 (3): $135-144$

4. Werren M, Alberti E (2008) Aneurisma del setto interatriale di origine familiare: quali implicazioni? G Ital Cardiol 9(8): 579-582.

5. Overell JR, Bone I, Lees KR (2000) Interatrial septal abnormalities and stroke: a meta-analysis of case-control studies. Neurology 55(8): 1172 1179 . 
6. Stroke Prevention and Educational Awareness Diffusion (SPREAD) Italian Guidelines for stroke. VIII Edition 2017

7. Messé SR, Silverman IE, Kizer JR, Homma S, Zahn C, et al. (2004) Practice Parameter: recurrent stroke with patent foramen ovale and atrial septal aneurysm: report of the Quality Standards Subcommittee of the American Academy of Neurology. Neurology 62(7): 1042-1050.

8. Hammerstingl C, Bauriedel B, Stüsser C, Momcilovic D, Tuleta I, et al (2011) Risk and fate of residual interatrial shunting after transcatheter closure of patent foramen ovale: a long term follow up study. Eur J Med Res 16(1): 13-19.

9. Hajsadeghi S, Chitsazan M, Namazi M, Chitsazan M (2012) An aneurysmal interatrial septum with a patent foramen ovale and multiple fenestrations closed by a single occluder device. Acta Med Iran 50(10): 713-716.

10. Rigatelli G, Dell'avvocata F, Cardaioli P, Braggion G, Giordan M, et al (2012) Long-term results of the amplatzer cribriform occlude for patent foramen ovale with associated atrial septal aneurysm: impact on occlusion rate and left atrial functional remodeling. Am J Cardiovasc Dis 2(1): 68-74.

11. Cerrato P, Priano L, Imperiale D, Bosco G, Destefanis E, et al. (2006) Recurrent cerebrovascular ischaemic events in patients with interatrial septal abnormalities: a follow-up study. Neurol Sci 26(1): 411-418.

12. Fisher M, McAllister M (2015) Cardiological Aspects of Stroke Prevention. Circ J 79(2): 271-277.

13. George PM, Albers GW (2014) Aortic arch atheroma. a plaque of a different color or more of the same? Stroke 45(5): 1239-1240.

14. Capmany RP, Ibañez MO, Pesquer XJ. (2010) Complex Atheromatosis of the Aortic Arch in cerebral infarction. Curr Cardiol Rev 6(3): 184-193.

15. Alvarez C, Aslam HM, Wallach S, Mustafa MU (2018) A Large grade 5 mobile aortic arch atheromatous plaque: cause of cerebrovascular accident. Case Rep Med doi: 10.1155/2018/5134309.

16. Lee JS, Chandraratna PAN (2003) Images in clinical medicine. peripheral embolism from an aortic-arch atheroma.
17. Beckman JA, Gerhard-Herman M (2004) Peripheral embolism from an aortic-arch atheroma. N Engl J Med 350(14): 1472.

18. Zavala JA, Amarrenco P, Davis SM, Jones EF, Young D, et al. (2006) Aortic arch atheroma. Int J Stroke 1(2): 74-80.

19. Cohen A, Tzourio C, Bertrand B, Chauvel C, Bousser MG, et al. (1997) Aortic plaque morphology and vascular events: a follow-up study in patients with ischemic stroke. FAPS Investigators. French Study of Aortic Plaques in Stroke. Circulation 96(11): 3838-3841.

20. Fujimoto S, Yasaka M, Otsubo R, et al. (2004) Aortic arch atherosclerotic lesions and the recurrence of ischemic stroke. Stroke 35(6): 1426-1429.

21. Weinberger J (2002) Noninvasive imaging of atherosclerotic plaque in the arch of the aorta with transcutaneous B-mode ultrasonography. Neuroimaging Clin N Am 12(3): 373-380.

22. Gottsegen JM, Coplan NL (2008) The Atherosclerotic aortic arch: considerations in diagnostic imaging. Prev Cardiol 11(3): 162-167.

23. Ryoo S, Chung JW, Lee MJ, Kim SJ, Lee JS, et al. (2016) An Approach to working up cases of embolic stroke of undetermined source. J Am Heart Assoc 5(3): e002975.

24. Amarenco P, Cohen A, Tzourio C, Bertrand B, Hommel M, et al. (1994) Atherosclerotic disease of the aortic arch and the risk of ischemic stroke. N Engl J Med 331(22): 1474-1479.

25. Sen S, Hinderliter A, Sen PK, Simmons J, Beck J, et al. (2007) Aortic arch atheroma progression and recurrent vascular events in patients with stroke or transient ischemic attack. Circulation 116(8): 928-935.

26. Kong Q Ma X (2015) Contributing mechanisms of aortic atheroma in ischemic cerebrovascular disease. J Stroke Cerebrovasc Dis 24(12): 2653-2659.

27. Amarenco P, Davis S, Jones EF, Cohen AA, Heiss WD, et al. (2014) Clopidogrel plus aspirin versus warfarin in patients with stroke and aortic arch plaques. Stroke 45(5): 1248-1257. 